\title{
Intermittent negative pressure wound therapy with instillation for the treatment of persistent periprosthetic hip infections: a report of two cases
}

Mehmet Salih Söylemez'

Korhan Özkan ${ }^{2}$

Bülent Kılıç3

Samet Erinç ${ }^{4}$

'Department of Orthopaedics and Traumatology, Bingöl State Hospital, Bingöl, ${ }^{2}$ Department of Orthopaedics and Traumatology, Faculty of Medicine, Medeniyet University, Istanbul, ${ }^{3}$ Department of Orthopaedics and Traumatology, Orthopaedic Surgery Clinic, Istanbul Gelișim University, Tekirdağ, ${ }^{4}$ Department of Orthopaedics and Traumatology, Istanbul Medeniyet University, Göztepe Training and Research Hospital, Istanbul, Turkey
Correspondence: Mehmet Salih Söylemez Department of Orthopaedics and Traumatology, Bingöl State Hospital, Kültür Mahallesi, Oğuzhan Caddesi, No 22, Bingöl, Turkey 12000 Email slhsylmz@gmail.com

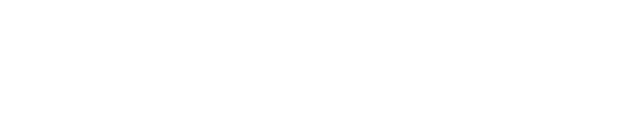

\begin{abstract}
Intermittent negative pressure wound therapy with instillation (NPWTi) is starting to be used successfully to treat early periprosthetic infections of endoprostheses. However, few articles have reported the outcome of treatment with intermittent NPWTi for late persistent periprosthetic infections of the hip. In this study, we report two cases who underwent several rounds of radical wound debridement for the treatment of a late persistent periprosthetic infection of the hip. Intermittent NPWTi was used in both cases. Patients were treated successfully and there was no recurrence after 3 and 1 years of follow-up, respectively.
\end{abstract}

Keywords: negative pressure, vacuum-assisted, periprosthetic infection, hip

\section{Introduction}

The use of topical negative pressure for a prolonged period to promote the debridement and healing of wounded tissue was first described by Fleischmann et $\mathrm{al}^{1}$ in 1993. Since then, various methods and devices have been used to facilitate its use in clinical practice. ${ }^{2-4}$ The development of automated, controlled commercially available systems (ie, vacuum-assisted closure [VAC]) has enabled the delivery of continuous or intermittent negative pressure to wounded tissues. ${ }^{5}$

VAC has been used to treat almost every type of wound encountered in orthopedic surgery. In particular, its use in early periprosthetic infections of the hip and knee has been successful. ${ }^{6,7}$ Classically, VAC creates a barrier and negative pressure for an extended period to promote the removal of excess fluid and debris. It decreases tissue bacterial levels and increases tissue perfusion, enhances granulation tissue formation, and improves wound healing. ${ }^{8,9}$ Unlike classic VAC, intermittent negative pressure wound therapy with instillation (NPWTi) enables the instillation of local antibiotics and/or antiseptics using an additional irrigation system. The amount and duration of the pressure can be determined by adjusting the fluid flow.

Intermittent NPWTi is starting to be used successfully to treat early periprosthetic infections of endoprostheses. ${ }^{10,11}$ However, few articles have reported the outcome of treatment with intermittent NPWTi for late persistent periprosthetic infections of the hip. ${ }^{12,13}$ In this study, we report two cases who underwent several rounds of radical wound debridement for the treatment of a late persistent periprosthetic infection of the hip. Intermittent NPWTi was used in both cases. The patients were treated successfully, and there was no recurrence after 3 and 1 years of follow-up, respectively. Ethical permission from a review board was unnecessary because treatment modalities 
performed in this study were accepted by both patients and written informed consent was given. This study complied with the guidelines from the Declaration of Helsinki.

\section{Case I}

The patient was a 52-year-old woman with a history of asthma and systemic lupus erythematosus. The patient had undergone bilateral total hip arthroplasty for right and left hip osteoarthritis at a different institution 7 and 8 years, respectively, before visiting our hospital. The patient was referred to our institution for aseptic femoral component loosening of the left hip. This was revised successfully, but unfortunately required a two-stage revision 1 year later because of septic loosening.

Over the next 2 years, one more two-stage revision arthroplasty was performed (Figure 1A). However, the infection again recurred, so finally all of the hardware was removed and radical debridement using various antibiotic-containing spacers was performed. This procedure was performed five more times.

During all five sessions, obvious sequestra and dead bone were removed, and the bone was resected to bleeding osseous tissue to ensure viability. Scarred ischemic or necrotic subcutaneous tissues, deep fascia, and muscle were also excised to obtain a healthy bleeding tissue bed.
Cultures taken from the deep tissue at the time of surgery revealed Candida ssp. once, Morganella morganii once, Staphylococcus epidermidis four times, Acinetobacter baumannii three times, and vancomycin-resistant enterococci three times. Amphotericin B-, teicoplanin-, vancomycinand gentamycin-containing cement, and finally cementcontaining daptomycin powder was prepared and used. Parenteral sulfamethoxazole-trimethoprim, posaconazole, linezolid, ceftriaxone, teicoplanin, imipenem, and daptomycin were added to the treatment protocol based on the culture results. However, sinus tracts occurred after the surgeries (Figure 1B). The duration of hospital stay was 22 months.

At the time of removal of the last spacer, meticulous debridement was performed and all ischemic and infected bone and soft tissue were excised. All open bone wounds were then filled using polyurethane sponges and an airtight seal of the VAC dressing (Ferah Medical, Istanbul, Turkey) was maintained. One tube was applied proximally to the sponges for irrigation and one tube was applied to the middle of the wound for negative pressure. The wound was left open.

A negative pressure of $180 \mathrm{mmHg}$ was applied initially. After 24 hours, the pressure was decreased to $120 \mathrm{mmHg}$. In each cycle, after 10 minutes, brake suction was added to the wound over a 30-minute period. Intravenous ceftriaxone was
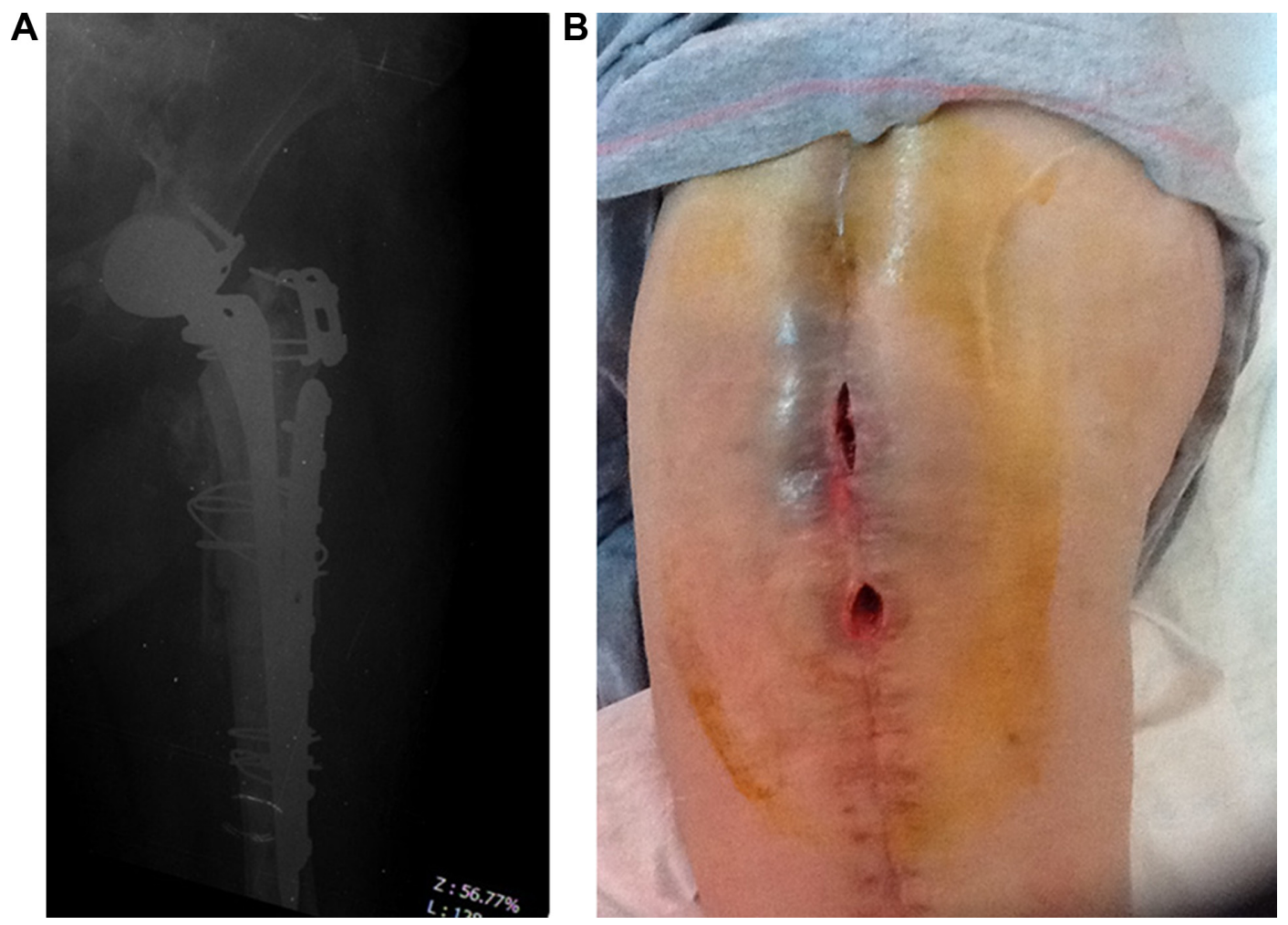

Figure I (A) Radiological appearance of the patient after the second revision arthroplasty with a proximal femoral allograft. (B) Clinical appearance of the patient before the final operation (case I). 

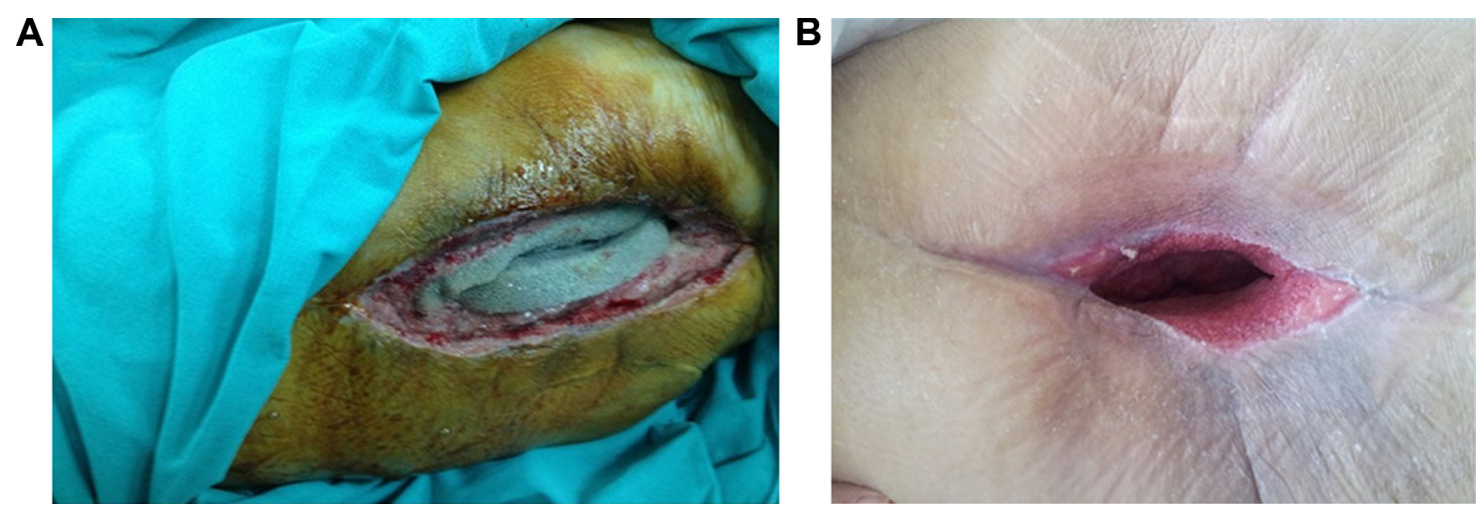

Figure 2 Wound appearance at 2 weeks (A) and 7 weeks (B) following the operation (case I).

started and $500 \mathrm{mg}$ of daptomycin in 1,000 $\mathrm{cc}$ of saline was applied over an 18-hour period through the irrigation system. The wound was irrigated with 1,000 cc of diluted Batikon during the remaining 6 hours during the first 2 days. Cultures revealed $A$. baumannii. Ceftriaxone was thus changed to daptomycin and ampicillin-sulbactam.

The first four changes of VAC were performed once per week under general anesthesia because of the open wound (Figure 2A). After the first month, VAC was changed every 4 days without disturbing the granulation tissue. This allowed inspection of the wound to evaluate the rate of ingrowth of granulation tissue.

After 15 days, wound cultures showed no further evidence of infection, good granulation tissue was observed, and the drained fluid was serous. Irrigation with local antibiotic application was stopped at that point. The erythrocyte sedimentation rate and C-reactive protein (CRP) level returned to normal after 28 days. Parenteral antibiotic treatment was stopped after 4 weeks, and an enteral antibiotic was administered during the next 3 weeks.

Skin cover was achieved through spontaneous epithelialization of granulation tissue after 9 weeks (Figure 2B). The patient refused placement of a new endoprosthesis and was mobilized using an ischium support brace after 9 weeks of hospital stay.

The patient was followed in the outpatient department at intervals of 3-6 weeks during the first 6 months for clinical and radiological evaluation to assess recurrence. No recurrence had been detected after 3 years of follow-up. No further prosthesis was implanted.

\section{Case 2}

The patient was a 65-year-old man with a history of hypertension. The patient had previously undergone an operation because of fracture of the neck of the left femur.
Osteosynthesis with cannulated screws was performed at a different institution. After 2 years, the patient had developed osteoarthritis for which he underwent cementless total hip arthroplasty. Ten years postoperatively, the patient developed aseptic loosening of the acetabular cup with protrusion into the pelvis and was referred to our clinic (Figure 3). Singlestage revision arthroplasty was scheduled.

During the operation, all prosthesis components were removed. The defective acetabulum was reconstructed using a spongious chip allograft and a flanged acetabular reconstruction cup (CONTOUR; Smith and Nephew, Memphis, TN, USA) was implanted with screws. Vancomycin-gentamycin embedded cement was used to fix the acetabular polar cup into the acetabular reconstruction cup. A cementless pressfit femoral component was used for the femoral revision (Figure 4A). Unfortunately, extensive drainage started 3 days postoperatively with increased fever. His CRP levels and leukocyte count continued to increase for the next 10 days,

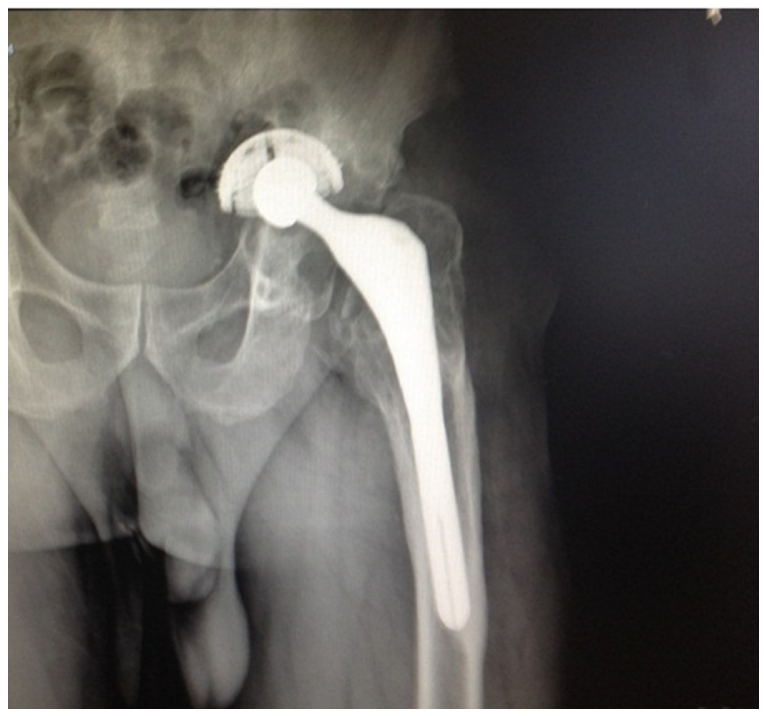

Figure 3 Preoperative $X$-ray of the patient (case 2). 
A

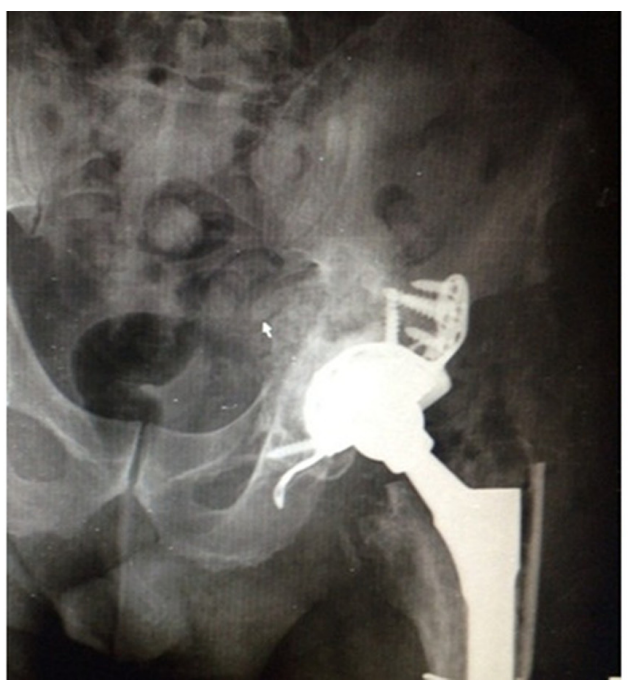

B

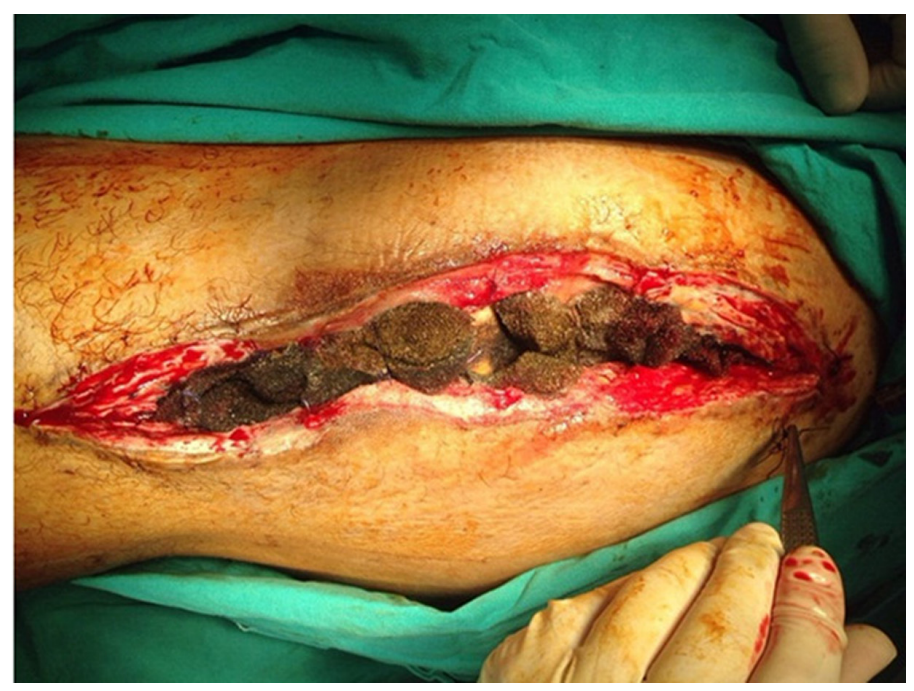

Figure 4 (A) Postoperative X-ray of the patient. (B) Wound appearance at the time of sponge removal (case 2).

so the hip was debrided with irrigation and the acetabular cup was replaced with a new one. His culture result was consistent with methicillin-resistant Staphylococcus aureus and teicoplanin was started. Ten days later, his fever recurred with further drainage. The patient underwent debridement with jet lavage again, which ultimately failed 12 days postoperatively when the drainage was recommended. Before removing the implants, NPWTi was used as a last possible therapeutic approach.

After meticulous debridement with jet lavage, all of the open wounds were filled using polyurethane sponges. One tube was applied proximally into the sponges for irrigation and another was applied distally into the sponges for the application of intermittent negative pressure (V.A.C. VeraFlo ${ }^{\mathrm{TM}}$ Kinetic Concepts, San Antonio, TX, USA). The wound was closed by taking care of the soft tissue layers.

A negative pressure of $200 \mathrm{mmHg}$ was applied initially. After 24 hours, the pressure was decreased to $125 \mathrm{mmHg}$. After 10 minutes, brake suction was added to the wound over a 15-minute period. Intravenous ceftriaxone was started. The wound was irrigated with $1,000 \mathrm{cc}$ of diluted Povidone-iodine and Prontosan (B. Braun Medical, Melsungen, Germany) over the first 2 days. Ceftriaxone ( $2 \mathrm{~g}$ ) in 1,000 cc of saline was added to the wound via the irrigation system during the first 5 days. Cultures revealed methicillin-resistant $S$. aureus, so the parenteral and local ceftriaxone were replaced with teicoplanin.

After 8 days, the drained fluid was serous in nature. After 15 days, the erythrocyte sedimentation rate and CRP level were within normal ranges. Irrigation with local antibiotic application was stopped at that point. After 2 weeks, the sponges were removed under general anesthesia. At this time, the wound was clean and well granulated, and was closed without disturbing the newly formed granulation tissue (Figure 4B). Parenteral antibiotic treatment was used for 3 weeks, after which an enteral antibiotic was administered for 3 weeks.

The patient was followed for recurrence with clinical and radiological evaluations in the outpatient department at intervals of 3-6 weeks during the first 6 months. No recurrence was noted during 1 year of follow-up.

\section{Discussion}

Intermittent NPWTi is indicated for patients who would benefit from continuous negative-pressure wound therapy (NPWT) as well as the controlled delivery and vacuumassisted drainage of topical wound treatment solutions and suspensions, including wound cleansers, over the wound bed. ${ }^{14}$ Intermittent NPWTi is composed of three phases: instillation of local antiseptics or antibiotics, followed by a delay to allow the antibiotics to soak and the application of negative pressure to the wound for the suction of excess fluid and debris.

Although the physiological mechanism remains obscure, fluid administration and intermittent NPWT has been shown to be effective in terms of wound healing. ${ }^{15}$ Borgquist et al ${ }^{9}$ reported that intermittent NPWT promoted increased blood flow, which is known to facilitate oxygenation and nutrient supply, stimulating angiogenesis and granulation tissue formation. Using porcine excisional wounds, Lessing et a ${ }^{15}$ showed that saline (the type of fluid can vary according to wound type) administration during NPWT resulted in superior and more rapid filling of wounds with granulation tissue. 
Although periprosthetic infections of the hip and knee are seen in only $1 \%$ of patients, they are the main complications of arthroplasty. In early infections, the implant can often be left in place with radical debridement and polyethylene replacement until 4 weeks after the operation. However, in late infections, in addition to radical debridement all prostheses must be revised to eradicate infection by either one- or two-stage revision. ${ }^{16}$ However, the rate of successful infection eradication after one- or two-stage revision arthroplasty is markedly higher than that after early periprosthetic infection. ${ }^{16}$

The successful use of intermittent NPWTi for early periprosthetic infections has been reported. Lehner and Bernd $^{10}$ treated two hip and one knee early periprosthetic infections successfully using intermittent NPWTi. Kirr et al ${ }^{11}$ reported the successful treatment of early knee and hip periprosthetic infections with intermittent NPWTi.

Few reports have addressed the efficacy of intermittent NPWTi for the treatment of late persistent periprosthetic infections. Riepe and Schneider ${ }^{12}$ reported the case of a 62-year-old patient with inflammation of the proximal femur 3 years after undergoing a total hip arthroplasty. The authors searched for an infection, but came to the conclusion that inflammation was causing the symptoms. The patient was treated successfully by intermittent NPWTi. In our first case, the patient had a history of systemic lupus erythematosus, which we believe worsened and contributed to superinfection of the wound.

Selecting an appropriate local antibiotic for use in cement spacers is important for revision arthroplasty. The gold standard for appropriate antibiotic selection is deep tissue culture results, which can take 4-14 days to become available. Vancomycin, tobramycin, and gentamycin are the most frequently used antibiotics in cement because of their broad spectrum of activity. ${ }^{16}$ However, not all bacteria can be successfully treated using these agents. As seen in our cases, cultures can result in the isolation of unexpected microorganisms. In the first case, Candida spp. were isolated in previous debridement sessions and A. baumannii during the final intervention. Daptomycin, which is used for the treatment of $A$. baumannii infections, is available as a powder and embedded in the cement. In the first case, vancomycin-resistant enterococci were isolated. These organisms are not susceptible to treatment with vancomycin-embedded cement, but respond to daptomycin. In addition, the parenteral antibiotic was changed to linezolid, which was added to the irrigation system at 5 days after the operation.
In conclusion, patients who had undergone several operations lost their soft tissue healing ability because of distorted vascularization and oxygenation, which results in persistent infections. Intermittent NPWTi has the following major advantages when compared with continuous NPWT: 1) instillation of antibiotics and local antiseptics to the wound, which promotes the eradication of microorganisms and accelerates granulation formation; and 2) the application of intermittent pressure to the wound enhances vascularization and granulation. Although large randomized controlled trials are needed to come to an exact conclusion and few studies of the use of intermittent NPWTi to treat late persistent periprosthetic hip infections have been published, we believe that this system may be useful for this condition. As shown by the two cases described here, intermittent NPWTi may be a simple and effective treatment alternative for persistent infections.

\section{Acknowledgment}

Written informed consent was obtained from the patients for publication of this manuscript and accompanying images.

\section{Disclosure}

The authors report no conflicts of interest in this work.

\section{References}

1. Fleischmann W, Strecker W, Bombelli M. Vacuum sealing as treatment of soft tissue damage in open fractures. Unfallchirurg. 1993;96: 488-492.

2. Mullner T, Mrkonjic L, Kwasny O, Vécsei V. The use of negative pressure to promote the healing of tissue defects: a clinical trial using the vacuum sealing technique. Br J Plast Surg. 1997;50:194-199.

3. Brock WB, Barker DE, Burns RP. Temporary closure of open abdominal wounds: the vacuum pack. Am Surg. 1995;61:30-35.

4. Fleischmann W, Russ M, Westhauser A, Stampehl M. Vacuum sealing as carrier system for controlled local drug administration in wound infection. Unfallchirurg. 1998;101:649-654.

5. Bovill E, Banwell PE, Teot L, et al. International Advisory Panel on Topical Negative Pressure. Topical negative pressure wound therapy: a review of its role and guidelines for its use in the management of acute wounds. Int Wound J. 2008;5(4):511-529.

6. Kelm J, Schmitt E, Anagnostakos K. Vacuum-assisted closure in the treatment of early hip joint infections. Int J Med Sci. 2009;6(5):241-246.

7. Lüdemann M, Haid S, Wülker N, Rudert M. Results of vacuum sealing therapy in joint infections. Z Orthop Ihre Grenzgeb. 2006;144(6): 602-608.

8. Banwell PE, Musgrave M. Topical negative pressure therapy: mechanisms and indications. Int Wound J. 2004;1(2):95-106.

9. Borgquist $\mathrm{O}$, Ingemansson R, Malmsjö M. The effect of intermittent and variable negative pressure wound therapy on wound edge microvascular blood flow. Ostomy Wound Manage. 2010;56(3):60-67.

10. Lehner B, Bernd L. V.A.C.-instill therapy in periprosthetic infection of hip and knee arthroplasty. Zentralbl Chir. 2006;131(Suppl 1):S160-S164.

11. Kirr R, Wiberg J, Hertlein H. Clinical experience and results of using the V.A.C. instill therapy in infected hip and knee prosthetics. Zentralbl Chir. 2006;131 (Suppl 1):S79-S82. 
12. Riepe G, Schneider M. V.A.C. instill - first experiences on an inflammatory process of the proximal femur 3 years after hip-joint operation. Zentralbl Chir. 2006;131(Supp1 1):S157-S159.

13. Moch D, Fleischmann W, Westhauser A. Instillation vacuum sealingreport of initial experiences. Langenbecks Arch Chir Suppl Kongressbd. 1998;115:1197-1199.

14. Wolvos T. The use of negative pressure wound therapy with an automated, volumetric fluid administration: an advancement in wound care. Wounds. 2013;25(3):75-83.
15. Lessing MC, James RB, Ingram SC. Comparison of the effects of different negative pressure wound therapy modes - continuous, noncontinuous, and with instillation - on porcine excisional wounds. Eplasty. eCollection. 2013;13:e51

16. Fink B. Revision of late periprosthetic infections of total hip endoprostheses: pros and cons of different concepts. Int J Med Sci. 2009; 6(5):287-295.

\section{Publish your work in this journal}

Therapeutics and Clinical Risk Management is an international, peerreviewed journal of clinical therapeutics and risk management, focusing on concise rapid reporting of clinical studies in all therapeutic areas, outcomes, safety, and programs for the effective, safe, and sustained use of medicines. This journal is indexed on PubMed Central, CAS,
EMBase, Scopus and the Elsevier Bibliographic databases. The manuscript management system is completely online and includes a very quick and fair peer-review system, which is all easy to use. Visit http://www.dovepress.com/testimonials.php to read real quotes from published authors.

Submit your manuscript here: http://www.dovepress.com/therapeutics-and-clinical-risk-management-journal 